\title{
PAIN AFTER HERNIA SURGERY MUST BE AVOIDED AND HOPEFULLY TREATED
}

\author{
(C) Springer-Verlag 2014
}

\section{P5:04}

\author{
QUICK HOT SPOT: SYSTEMATIC \\ PATHOLOGICAL ASSESSMENT OF EXPLANTED \\ HERNIA MESHES REVEALS IMPORTANT \\ INFORMATION OF MESH-BODY INTERACTIONS \\ V Iakovlev $^{1}$, G Iakovleva ${ }^{2}, R_{\text {Bendavid }}^{3}$ \\ ${ }^{\prime}$ St, Michael's Hospital, University of Toronto, Toronto, CANADA \\ ${ }^{2}$ Markham Stouffille Hospital, Markham, CANADA \\ ${ }^{3}$ Shouldice Hospital, Richmond Hill, CANADA
}

Purpose: Polypropylene meshes, introduced in the late 50's, spread to other anatomical sites over the decades of use and presently are employed in millions of surgeries worldwide. Up to $10 \%$ of meshes are excised for complications or recurrence generating a large, but underutilized pool of study material. There is a surprising lack of studies based on the meshes explanted from the patients. Paradoxically, in the presence of abundant material from humans most conclusions of mesh-body interactions are based on animal studies. We aimed to standardize pathological examination of explanted meshes and correlate histological findings with clinical presentations.

Methods: Consecutive specimens of explanted polypropylene hernia meshes received at the pathology department within last two years were examined according to a predefined protocol. Out of 32 available specimens 13 were ventral and 19 inguinal hernia meshes. Median age was 61 years (range 24-82), 12 female and 20 male patients. For ventral hernias 2 were removed for migration into the bladder and fallopian tube, 4 for infection and 7 for recurrence. For groin meshes 7 were excised for pain and 12 for recurrence. The following microscopic parameters were assessed: mesh deformation, extent of fibrous encapsulation, nerve density and ingrowth, degree and type of inflammation, edema, vascular thrombosis, fat necrosis, attachment to muscle, and polypropylene degradation. Subjective parameters were scored independently by two pathologists. Two-tailed T-test and Pearson correlation were calculated to assess the significance of associations.

Results: In relation to reason for excision, the most significant finding was higher density of nerve branches ingrown into the meshes removed for pain compared to those removed for other complications $(p<0.001)$. Connection with striated muscle showed less pronounced, but significant association with pain $(p=0.05)$. Meshes removed for pain also showed trends of more exuberant foreign body type inflammation $(p=0.15)$ and edema in the scar inhabiting the mesh $(p=0.12)$. The amount of scarring with bridging fibrosis and the degree of foreign body reaction tended to be reduced in meshes with a longer period since implantation (correlation $=-0.4$ for both parameters). Polypropylene material showed a layer of degradation forming a sheath around the mesh filaments. The layer was detectable by its ability to trap histological dyes in the microcracks and become stained in the microscopic sections, however the stainable layer retained optical properties of polypropylene in polarized light.

Conclusions: We observed several important findings and associations related to mesh complications. One of the main findings related to pain is innervation within the tissue ingrown into the mesh. The innervated tissue is exposed to all regular pain mechanisms including nerve entrapment, compression by edema, inflammatory and other mechanisms. Histologically, mechanical nerve irritation of entrapped nerves is likely the leading mechanism while connection to striated muscle, edema and foreign body reaction appear to contribute in the development of pain. In relation to the body reaction to the mesh, the extent of scarring and foreign body inflammation tend to become reduced over time, however the change is mild. Knitted polypropylene meshes remain encapsulated in scar and retain the ability to trigger foreign body reaction many years after implantation. We also found that polypropylene used in mesh manufacturing degrades while exposed to the body environment and the clinical impact of polypropylene degradation needs to be studied further. Overall, the explant specimens, mostly neglected over the decades contain primary information of the mesh-body interactions. A standardized systematic examination of explanted mesh specimens can help our understanding of the mechanisms of complications and build a body of knowledge needed for further technical developments and treatment strategies in the field of hernia repair.

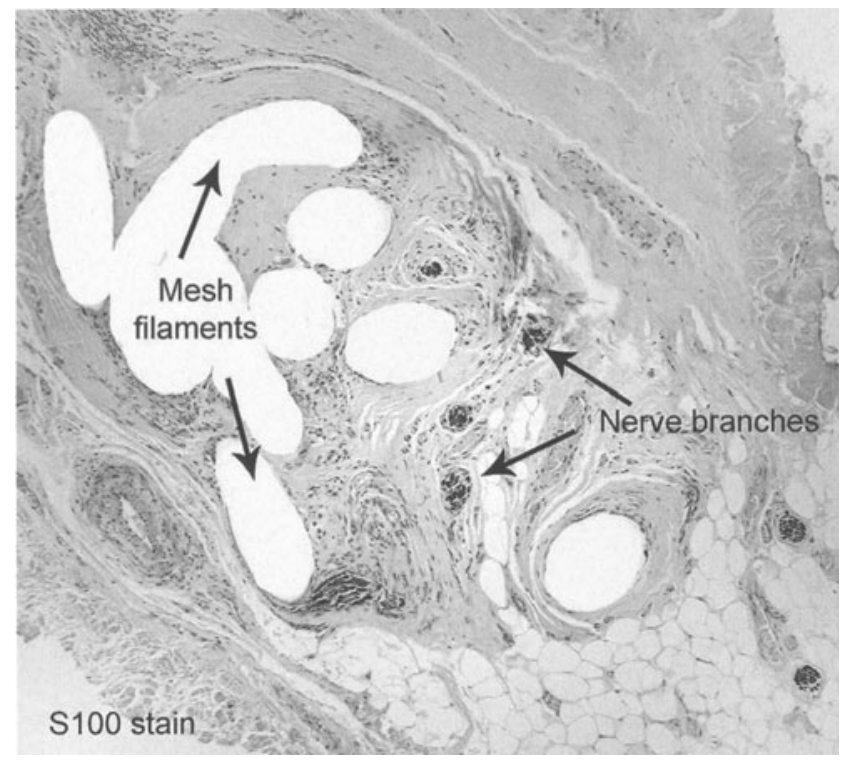

